\title{
A Feynman-Kac Formula for the Quantum Heisenberg Ferromagnet. II
}

\author{
H. Hogreve ${ }^{1}$, W. Müller ${ }^{2}$, J. Potthoff ${ }^{3}$, and R. Schrader ${ }^{4}$ \\ ${ }^{1}$ Hahn-Meitner Institut Berlin, D-1000 Berlin \\ ${ }^{2}$ Akademie der Wissenschaften der DDR, Berlin, German Democratic Republic \\ ${ }^{3}$ Universität Bielefeld, Bielefeld, Federal Republic of Germany, and LSU at Baton Rouge, USA \\ ${ }^{4}$ Freie Universität Berlin, D-1000 Berlin
}

\section{Dedicated to Res Jost and Arthur Wightman}

\begin{abstract}
This article continues the analysis of the first arcticle under the same title. Using methods of stochastic analysis we prove Feynman-Kac formulas for the relevant heat kernels. We also present classical limit theorems.
\end{abstract}

This paper is the second part of a work devoted to a probabilistic approach for the quantum Heisenberg ferromagnet relating this model to a Euclidean lattice field theory.

In Sect. 2 and 3 of the previous article heat kernel representations of the partition function were given. In Sect. 4 the resulting Euclidean field theoretic Lagrangian was calculated. Here, in Sect. 5 and 6, we formulate Feynman-Kac representations for the heat kernels involved, first for the one-lattice point theory and then for the full interacting theory on an arbitrary finite lattice. Our presentation is strongly influenced by Bismut's work on probabilistic proofs of index theorems [Bi2].

In Sect. 7 we present classical limit theorems for the purely bosonic sector of the theory.

We use the notations and results of [HMPS].

\section{Feynman-Kac Formula for the One Lattice Point Theory}

In this section we will establish a rigorous stochastic expression for the kernel of the semigroup

$$
e^{-t\left\{\frac{1}{m} \bar{\square}-i d \pi^{2}(h)\right\}},
$$

where $t>0$ and $h \in \mathbf{g}$. For simplicity we consider the case $m=1$. The general case $m>0$ can be obtained by the rescaling $t \rightarrow \frac{t}{m}, h \rightarrow h m$. The first step is to construct the stochastic process on $\bar{\Lambda}\left(\mathscr{L}^{\lambda}\right)$ that is generated by the (horizontal) Bochner Laplacian $-\left(\nabla^{\lambda}\right)^{2}$. The stochastic representation of the kernel (5.1) will then be 
obtained by a variant of the well known subordination procedure used by Malliavin [Ma]. It consists in setting up a stochastic differential equation (SDE) whose solution takes account of the difference

$$
\bar{\square}-i d \pi^{\lambda}(h)+\frac{1}{2}\left(\phi^{\lambda}\right)^{2} .
$$

In fact, this is possible due to the Weitzenböck type formula (2.32) and relation (2.31). These equations imply that the expression (5.2) is a first order differential operator $-i \nabla_{K_{h}}^{\lambda}$, i.e. a "drift" term, plus a strict vector bundle endomorphism.

The construction of a stochastic motion in $\bar{\Lambda}\left(\mathscr{L}^{\lambda}\right)$ is facilitated by the fact that we have sufficiently many vector fields on $M_{\lambda}$ at our disposal, namely the Killing vector fields which span the tangent space at each point. In particular, writing $K_{Y}$ with $Y \in \mathbf{g}$ in local real coordinates $x^{j}$ for $z \in M_{\lambda}$ as

$$
K_{Y}=K_{Y}^{j} \frac{\partial}{\partial x^{j}}
$$

we have (see e.g. [KN, Vol. II, p. 200])

$$
-\sum_{\alpha, \beta} K_{Y_{\alpha}}^{i} K_{Y_{\beta}}^{j} b^{\alpha \beta}=g^{i j}
$$

for the metric on $M_{\lambda}$. Moreover, the vanishing of the covariant divergence of the Killing vector fields implies

$$
\sum_{\alpha, \beta} K_{Y_{\alpha}}^{i}\left(\frac{\partial}{\partial x^{i}} K_{Y_{\beta}}^{j}\right) b^{\alpha \beta}=g^{i k} \Gamma_{i k}^{j} .
$$

Therefore the Laplace-Beltrami operator $\Delta \geqq 0$ on $M_{\lambda}$ takes the form

$$
\Delta=\sum_{\alpha, \beta} K_{Y_{\alpha}} K_{Y_{\beta}} b^{\alpha \beta} .
$$

We rewrite these formulas in the following way. Let the matrix $d^{\alpha \beta}=d^{\beta \alpha}$ be the unique positive definite square root of $-b^{\alpha \beta}$ and define global vector fields $L_{\alpha}$ on $M$ by

Then (5.4) takes the form

$$
L_{\alpha}=\sum_{\beta} K_{Y_{\beta}} d^{\alpha \beta} .
$$

whereas (5.5) gives

$$
\sum_{\alpha} L_{\alpha}^{i} L_{\alpha}^{j}=g^{i j},
$$

such that

$$
\sum_{\alpha} L_{\alpha}^{i}\left(\frac{\partial}{\partial x^{i}} L_{\alpha}^{j}\right)=-g^{i k} \Gamma_{i k}^{j}
$$

$$
-\sum_{\alpha} L_{\alpha}^{2}=\Delta \text {. }
$$

Consider the principal $U_{\lambda}$ bundle $\mathscr{P}$ over $M_{\lambda}$ defined as

$$
\begin{aligned}
U_{\lambda} \rightarrow & G \\
& \downarrow^{\pi} \\
& G / U_{\lambda} \cong M_{\lambda} .
\end{aligned}
$$


It is easy to see that $\bar{\Lambda}\left(\mathscr{L}^{\lambda}\right)$ is the bundle associated to $\mathscr{P}$ via the following representation $\sigma$ of $U_{\lambda}$. A typical fibre of $\bar{\Lambda}\left(\mathscr{L}^{\lambda}\right)$ is provided by the vector space

$$
\mathscr{V}=\mathbb{C} \otimes \Lambda^{*} T_{\lambda}^{*(0,1)} M_{\lambda} .
$$

Let $\sigma=\sigma_{1} \otimes \sigma_{2}$, where $\sigma_{1}(u)=\chi_{\lambda}(u)$ with $u \in U_{\lambda}$ and where $\sigma_{2}$ is the isotropy representation of $U_{\lambda}$ on $\Lambda^{*} T_{\lambda}^{*(0,1)} M_{\lambda}$ resulting from the coadjoint action of $G$ on $\mathrm{M}_{\lambda}$. Let $\omega^{\lambda}$ be the connection form on $\mathscr{P}$ associated to the connection $\nabla^{\lambda}$. By $\widetilde{X}$ we denote the horizontal lift to $\mathscr{P}$ (via $\omega^{\lambda}$ ) of a vector field $X$ on $M_{\lambda}$. In particular we have $d \pi\left(\widetilde{K}_{Y}\right)=K_{Y}$ (note that $\widetilde{K}_{Y} \neq Y$ where we identify $Y \in \mathbf{g}$ with the left invariant vector field on $G$ generated by it), $d \pi\left(\tilde{L}_{\alpha}\right)=L_{\alpha}$ and due to (5.10)

$$
-\sum_{\alpha} \tilde{L}_{\alpha}^{2}(f \circ \pi)=(\Delta f) \circ \pi
$$

for $f \in C^{\infty}\left(M_{\lambda}\right)$. Let $w(\cdot)$ be a standard Brownian motion on $\mathbb{R}^{\operatorname{dim} G}$ and consider the following SDE on $\mathscr{P}$ :

$$
d u(t)=\sum_{\alpha} \widetilde{L}_{\alpha}(u(t)) d w^{\alpha}(t) .
$$

Here and in the sequel we adopt the convention of e.g. [Bi2] and denote the Stratonovich differential of a process $Z$ by $d Z$, its Itô-differential by $\delta Z$. Since $\mathscr{P}$ is compact and $\widetilde{L}_{\alpha}$ horizontal, standard theorems (see e.g. [IW]) guarantee existence and uniqueness of solutions to (5.13) with initial conditions $u(0)=u \in \mathscr{P}$. Also $z(t)=\pi u(t)$ defines for $t \geqq 0$ a standard Brownian motion on $M$ starting at $z(0)=z=\pi u$. This follows immediately by projection of (5.13) yielding

$$
d z(t)=\sum_{\alpha} L_{\alpha}(z(t)) d w^{\alpha}(t),
$$

and then using standard stochastic calculus (cf. [IW]) combined with relation (5.10). Moreover, the process $u(t)$ on $\mathscr{P}$ with $t \geqq 0$ is related to the Bochner Laplacian- $\left(\nabla^{\lambda}\right)^{2}$ on $\bar{\Lambda}\left(\mathscr{L}^{\lambda}\right)$ in the following way. Via $\sigma$ the process $\{u(t), t \geqq 0\}$ may be considered (a.s.) as giving a map of the typical fibre $\mathscr{V}$ of $\bar{\Lambda}\left(\mathscr{L}^{\lambda}\right)$ onto the fibre $\pi^{-1}(z(t))$ (see e.g. $\left[\mathrm{KN}\right.$, Vol. I, p. 115]) and therefore $\tau_{0}^{t} \stackrel{\text { def }}{=} u u(t)^{-1}$ is the stochastic parallel transport of the fibre at $z(t)$ onto the fibre at $z$.

Proposition 5.1. For all $t>0, \varphi \in C^{\infty}\left(\bar{\Lambda}\left(\mathscr{L}^{\lambda}\right)\right)$ and $z \in M_{\lambda}$

$$
\left(e^{\frac{t}{2}\left(\nabla^{2}\right)^{2}} \varphi\right)(z)=\mathbb{E}\left(\tau_{0}^{t} \varphi(z(t))\right) .
$$

Proof. We first note the relation

$$
d\left(\tau_{0}^{t} \varphi(z(t))\right)=\tau_{0}^{t} \sum_{\alpha}\left\{\nabla_{L_{\alpha}}^{\lambda} \varphi(z(t)) \delta w^{\alpha}(t)+\frac{1}{2}\left(\nabla_{L_{\alpha}}^{\lambda}\right)^{2} \varphi(z(t)) d t\right\},
$$

which is proved in [Bi1] for the case of a tensor bundle and with the frame bundle as associated principal bundle. The arguments in [Bi1], however, easily extend to the present case. Proposition 5.1 is then a consequence of (5.16) and the relation

$$
\sum_{\alpha}\left(\nabla_{L_{\alpha}}^{\lambda}\right)^{2}=\left(\nabla^{\lambda}\right)^{2}
$$

which is proved in the same way as (5.10). 
Let now $F$ be a smooth section of the bundle End $\left(\bar{\Lambda}\left(\mathscr{L}^{\lambda}\right)\right) \cong \bar{\Lambda}\left(\mathscr{L}^{\lambda}\right) \otimes \bar{\Lambda}\left(\mathscr{L}^{\lambda}\right)^{*}$ of strict vector bundle endomorphisms of $\bar{\Lambda}\left(\mathscr{L}^{\lambda}\right)$. Obviously this bundle is associated to $\mathscr{P}$ via the representation $\sigma \otimes \sigma^{*}$ of $U_{\lambda}$ on $\mathscr{V} \otimes \mathscr{V}^{*}$. If we denote the resulting stochastic parallel transport from the fibre over $z(t)$ to the fibre over $z$ by $\tilde{\tau}_{0}^{t}$, we have

$$
\tilde{\tau}_{0}^{t} F(z(t))=\tau_{0}^{t} F(z(t))\left(\tau_{0}^{t}\right)^{-1},
$$

which is an endomorphism of $\pi^{-1}(z)$. Moreover, for a vector field $X$ over $M_{\lambda}$ we again let $X^{*}$ be its dual 1 -form with respect to the metric $g$ on $M_{\lambda}$. We consider the SDE

$$
d U(t)=U(t)\left\{\tau_{0}^{t} F(z(t)) d t+i X^{*}(z(t)) \delta z(t)\right\}
$$

in the fibre $\pi^{-1}(z)$ of $\operatorname{End}\left(\bar{\Lambda}\left(\mathscr{L}^{\lambda}\right)\right)$ with initial condition $U(0)=$ id. Here the multiplication of the 1 -form $X^{*}$ by the Itô differential $\delta z$ is with respect to the LeviCivita connection on $M_{\lambda}$ in the sense of Meyer [Me]. In local real coordinates $x^{i}$ for $z$ with $X=X^{j} \partial / \partial x^{j}$,

$$
X^{*}(z(t)) \delta z(t)=X_{i}^{*}(z(t)) \delta x^{i}(t)+\frac{1}{2} g^{i j} \Gamma_{i j}^{k} X_{k}^{*}(z(t)) d t .
$$

With these preparations we are ready for the proof of the following result.

Theorem 5.2. Let $\varphi$ be a smooth section of $\bar{\Lambda}\left(\mathscr{L}^{\lambda}\right)$ and $t>0$. Then

$$
\left(e^{t\left\{\frac{1}{2}\left(\nabla^{\lambda}\right)^{2}+F+i \nabla_{x}^{\lambda}\right\}} \varphi\right)(z)=\mathbb{E}\left(U(t) \tau_{0}^{t} \varphi(z(t))\right) .
$$

Proof. We compute the differential of $U(t) \tau_{0}^{t} \varphi(z(t))$. By a well-known localization argument (see e.g. [IW]) we are allowed to work in local coordinates. Then

$$
\begin{aligned}
d(U(t) & \left.\tau_{0}^{t}(z(t))\right) \\
= & U(t)\left\{\left[\tilde{\tau}_{0}^{t} F(z(t))+\frac{i}{2} \sum_{\alpha}\left(L_{\alpha}^{i} \partial_{i} L_{\alpha}^{k} X_{k}^{*}\right)(z(t))+\frac{i}{2}\left(g^{i j} \Gamma_{i j}^{k} X_{k}^{*}\right)(z(t))\right] \tau_{0}^{t} \varphi(z(t))\right. \\
& \left.+\frac{1}{2} \tau_{0}^{t}\left(\left(\nabla^{\lambda}\right)^{2} \varphi\right)(z(t))+i \sum_{\alpha}\left(L_{\alpha}^{k} X_{k}^{*}\right)(z(t)) \tau_{0}^{t}\left(\phi_{L_{\alpha}}^{\lambda} \varphi\right)(z(t))\right\} d t \\
& + \text { martingale } \\
= & U(t) \tau_{0}^{t}\left\{(F \varphi)(z(t))+\frac{1}{2}\left(\left(\nabla^{\lambda}\right)^{2} \varphi\right)(z(t))+i\left(\nabla_{X}^{\lambda} \varphi\right)(z(t))\right\} d t \\
& + \text { martingale }
\end{aligned}
$$

where the equality (5.22) follows from standard stochastic calculus and (5.23) from relations (5.8) and (5.9).

As an application we make the choices $X=K_{h}$ and

$$
F(z)=i h(z)+i E_{h}(z)+\frac{1}{2} D \tilde{K}(z)-D S(z)-\frac{1}{2} \operatorname{tr} S(z)
$$

such that

$$
\bar{\square}-i d \pi^{\lambda}(h)=-\frac{1}{2}\left(\nabla^{\lambda}\right)^{2}-i \nabla_{K_{h}}^{\lambda}-F \text {. }
$$

In this way we obtain a Feynman-Kac formula for the semigroup (5.1) acting on smooth sections $\varphi$ of $\bar{\Lambda}\left(\mathscr{L}^{\lambda}\right)$.

Furthermore, we remark that the construction of the measure $d B_{z, z^{\prime}}^{t}$ of a Brownian bridge between $z$ and $z^{\prime}$ in $\mathrm{M}_{\lambda}$ in time $t$ may be taken over from Bismut 
[Bi3]. This measure lives on the space of continuous paths $z(\cdot)$ such that $z(0)$ $=z, z(t)=z^{\prime}$ and has the heat kernel of the Laplace-Beltrami operator as transition probability. If in (5.21) we then replace the expectation by $d B_{z, z^{\prime}}^{t}$ and $\varphi$ by 1 we arrive at a Feynman-Kac representation of the kernel of the semigroup (5.1).

Finally let us compare formula (5.21) for the operator (5.25) with the heuristic expressions (4.16) and (4.17). To this end let us pretend that $M_{\lambda}$ has global coordinates. Then the stochastic parallel transporter $\tau_{0}^{t}$ is formally given by

$$
\tau_{0}^{t}=\tau_{0}^{t}(z(\cdot))=\mathscr{P}\left(\exp \left\{\int_{0}^{t} \omega_{k}(z(s)) d x^{k}(s)\right\}\right),
$$

where $\omega=i A+C$ is the (local) connection form of the bundle $\bar{\Lambda}\left(\mathscr{L}^{\lambda}\right)$ (cf. Sect. 2).

Using stochastic calculus, we derive the following SDE:

$$
\begin{aligned}
d U(t) \tau_{0}^{t}= & U(t) \tau_{0}^{t}\left\{\left(i\left(K_{h}^{*}\right)_{k}(z(t))+\omega_{k}(z(t))\right) \delta x^{k}(t)\right. \\
& +\left[F(z(t))+\frac{i}{2}\left(g^{i j} \Gamma_{i j}^{k}\left(K_{h}^{*}\right)\right)(z(t))+i\left(\omega, K_{h}\right)(z(t))\right. \\
& \left.\left.+\frac{1}{2}\left(\omega, \omega^{*}\right)(z(t))+\operatorname{div} \omega(z(t))\right] d t\right\},
\end{aligned}
$$

where $F$ is as in (5.24). The formal solution of (5.27) satisfying the initial condition $U(t) \tau_{0 \mid t=0}^{t}=\mathrm{id}$ reads

with

$$
\begin{aligned}
U(t) \tau_{0}^{t}= & \mathscr{P}\left(\operatorname { e x p } \left\{\int _ { 0 } ^ { t } \left(N(z(s)) d s+i\left(K_{h}^{*}\right)_{k}(z(s)) \delta x^{k}(s)+\left(i A_{k}(z(s))\right.\right.\right.\right. \\
& \left.\left.\left.\left.+C_{k}(z(s))\right) d x^{k}(s)\right)\right\}\right)
\end{aligned}
$$

$$
\begin{aligned}
N(z)=i h(z) & -\frac{1}{2} \operatorname{tr} S(z)+i E_{h}(z)+\frac{1}{2} D \tilde{K}(z)-D S(z) \\
+ & \frac{1}{2}\left(K_{h}^{*}, K_{h}\right)(z)+\frac{i}{2}\left(g^{i j} \Gamma_{i j}^{k}\left(K_{h}^{*}\right)_{k}\right)(z) .
\end{aligned}
$$

This agrees with the formula (4.16) up to the free fermion Lagrangian there and the last term in (5.29) which is a correction due to the fact that the paths are (a.s.) nonrectifiable, and it ensures the correct transformation property of the Itô-integral under a change of coordinates. We recall that the free fermion Lagrangian combined with the Berezin integration serves to compute the fibrewise trace.

We conclude this section by giving a stochastic representation of the character of the irreducible representation $\pi_{\lambda}$. For this purpose $i d \pi_{\lambda}(h)$ in (5.1) has to be replaced by $d \pi_{\lambda}(h)$. We are thus led to the SDE

$$
d V(t)=V(t)\left\{\tilde{\tau}_{0}^{t} G(z(t)) d t+K_{h}^{*}(z(t)) \delta z(t)\right\}
$$

in the fibre $\pi^{-1}(z)$ of End $\left(\bar{\Lambda}\left(\mathscr{L}^{\lambda}\right)\right)$ with initial condition $V(0)=\mathrm{id}$. In $(5.30)$ $G \in C^{\infty}\left(\operatorname{End}\left(\bar{\Lambda}\left(\mathscr{L}^{\lambda}\right)\right)\right)$ is given as

$$
G(z)=h(z)+E_{h}(z)+\frac{1}{2} D \tilde{K}(z)-D S(z)-\frac{1}{2} \operatorname{tr} S(z) .
$$


Then the desired stochastic representation follows:

$$
\begin{aligned}
\operatorname{Trace}_{\mathscr{H}_{\lambda}}\left\{\pi_{\lambda}\left(e^{t h}\right)\right\} & =\operatorname{Trace}_{\mathscr{H}_{\lambda}}\left\{e^{t d \pi_{\lambda}(h)}\right\} \\
& =\operatorname{Trace}_{L^{2}\left(\bar{\Lambda}\left(\mathscr{L}^{\lambda}\right)\right)}\left\{\varepsilon e^{-t\left(m^{-1} \bar{\square}-d \pi^{\lambda}(h)\right)}\right\} \\
& =\int d \mu_{M_{\lambda}}(z) \int d B_{z, z}^{t} \operatorname{trace}_{\pi^{-1}(z)}\left\{\varepsilon V(t) \tau_{0}^{t}\right\},
\end{aligned}
$$

where $\operatorname{trace}_{\pi^{-1}(z)}$ denotes the trace in the fibre $\pi^{-1}(z)$ of End $\left(\bar{\Lambda}\left(\mathscr{L}^{\lambda}\right)\right)$ over $z$.

\section{Feynman-Kac Formula for the Interacting Case}

In this section we extend the methods of Sect. 5 to a general lattice $\Lambda$ and $J \neq 0$. Again we choose $m=1$ since the general case can be obtained by a rescaling argument. Hence $J$ is assumed to be sufficiently small. Since the interaction term $H_{A}^{\lambda}$ is a second order differential operator, we have to find a second order elliptic operator whose leading symbol agrees with that of $\bar{\square}_{\lambda}+H_{\Lambda}^{\lambda}$ and for which it is possible to associate a stochastic process. To construct such an operator, we first note that the metric $\hat{g}$ on $M_{A}$ introduced in Sect. 4 may be obtained in the following alternative way.

Let $A=(\alpha, k), B=\left(\beta, k^{\prime}\right), \ldots$ with $1 \leqq \alpha, \beta \leqq \operatorname{dim} G$ and $k, k^{\prime} \in \Lambda$ denote double indices. For given $J$ and $c=\left\{c^{\alpha \beta}\left(k, k^{\prime}\right)\right\}$ we define the matrix $D$ by

$$
D^{A B}=\left\{\begin{array}{ll}
b^{\alpha \beta}, & \text { if } k=k^{\prime} \\
2 J c^{\alpha \beta}\left(k, k^{\prime}\right), & \text { if } k \neq k^{\prime}
\end{array} .\right.
$$

For $J$ sufficiently small the hermitian matrix $-D$ will be positive definite; let $(-D)^{1 / 2}$ be its unique positive square root.

We define global vector fields on $M_{\lambda, \Lambda}$,

Then with

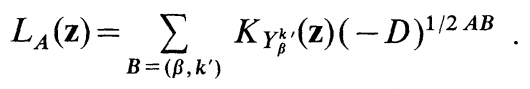

$$
L_{A}(\mathbf{z})=\sum_{\mu} L_{A}^{\mu}(\mathbf{z}) \frac{\partial}{\partial \mathbf{x}^{\mu}}
$$

in real local coordinates $\mathbf{x}^{\mu}$ for $z \in M_{\lambda, \Lambda}$, by construction we have

$$
\sum_{A} L_{A}^{\mu} L_{A}^{v}(\mathbf{z})=\hat{g}^{\mu v}(\mathbf{z})
$$

Next we introduce the principal $\times_{k \in \Lambda} U_{\lambda}$-bundle $\mathscr{P}_{\Lambda}=\times_{k \in \Lambda} \mathscr{P}=\times_{k \in \Lambda} G=G_{\Lambda}$ over $M_{\lambda, \Lambda}=\times_{k \in \Lambda} M_{\lambda, k}$ with projection $\pi$ and associated to $\bar{\Lambda}\left(\mathscr{L}_{\Lambda}^{\lambda}\right)$ via $\sigma_{\Lambda}$ given by $\sigma_{\Lambda}(\mathbf{u})=\bigotimes_{k \in \Lambda} \sigma(u(k))$ on the typical fibre of $\bar{\Lambda}\left(\mathscr{L}_{\Lambda}^{\lambda}\right) \cong \bigotimes_{k \in \Lambda} \mathscr{V}$ for $\mathbf{u}=\{u(k)\}_{k \in \Lambda} \in$ $\times_{k \in \Lambda} U_{\lambda}$ (cf. Sect. 5). Also let $\omega_{\Lambda}^{\lambda}$ be the connection form on $\mathscr{P}_{\Lambda}$ associated to the connection $\nabla_{\Lambda}^{\lambda}$ on $\bar{\Lambda}\left(\mathscr{L}_{\Lambda}^{\lambda}\right)$. Again $\tilde{X}$ denotes the horizontal lift (via $\omega_{\Lambda}^{\lambda}$ ) to $\mathscr{P}_{\Lambda}$ of a vector field $X$ on $M_{\lambda, \Lambda}$. In particular we have $d \pi\left(\tilde{L}_{A}\right)=L_{A}$. Let now $w(\cdot)$ be a standard Brownian motion on $\mathbb{R}^{\operatorname{dim} G|\Lambda|}$ and consider the following $\operatorname{SDE}$ on $\mathscr{P}_{A}$ :

$$
d u(t)=\sum_{A} \tilde{L}_{A}(u(t)) d w^{A}(t)
$$

which again has a unique solution for the initial condition $u(0)=u \in \mathscr{P}_{\Lambda}$. 
Also for nonnegative times $\mathbf{z}(t)=\pi(u(t))$ is Brownian motion on $M_{\lambda, A}$ with infinitesimal generator- $\sum_{A} L_{A}^{2}$ and starting point $\mathbf{z}=\pi(u)$. Note that $L_{A}$ is not a Killing vector field with respect to the metric $\hat{g}$. Hence this operator is not the Laplace-Beltrami operator for the metric $\hat{g}$ unless $J=0$. As in Sect. 5 we define $\tau_{0}^{t}$ $=u u(t)^{-1}$ to be the stochastic parallel transport in $\bar{\Lambda}\left(\mathscr{L}_{\Lambda}^{\lambda}\right)$ along $\mathbf{z}(\cdot)$ of the fibre at $\mathbf{z}(t)$ onto the fibre at $\mathbf{z}$.

If we set

$$
0 \leqq \hat{\Delta}=-\sum_{A} \nabla_{A L_{A}}^{\lambda} \nabla_{\Lambda L_{A}}^{\lambda},
$$

then in analogy to Proposition 5.1 we have

Proposition 6.1. For all $t>0, \varphi \in C^{\infty}\left(\bar{\Lambda}\left(\mathscr{L}_{\Lambda}^{\lambda}\right)\right)$ and $\mathbf{z} \in M_{\lambda, \Lambda}$,

$$
\left(\exp \left(-\frac{t}{2} \hat{\Delta}\right) \varphi\right)(\mathbf{z})=\mathbb{E}\left(\tau_{0}^{t} \varphi(\mathbf{z}(t))\right) .
$$

To obtain a stochastic representation for the kernel of the semigroup defined by the operator of our theory, we observe that by construction the difference

$$
\bar{\square}_{A}+H_{\Lambda}^{\lambda}-\frac{1}{2} \hat{\Delta}
$$

is a first order differential operator. In order to exhibit its structure we have to generalize the notion of covariant derivative in the following way. form

Consider an element $W$ of $C^{\infty}\left(\right.$ End $\left.\bar{\Lambda}\left(\mathscr{L}_{\Lambda}^{\lambda}\right) \otimes T_{\mathbb{C}} M_{\lambda, A}\right)\left(\supseteq C^{\infty}\left(T_{\mathbb{C}} M_{\lambda, \Lambda}\right)\right)$ of the

$$
W=\sum_{l=1}^{N} F_{l} \otimes X_{l} .
$$

Since $\nabla_{A X}^{\lambda}$ is $C^{\infty}\left(M_{\lambda, \Lambda}\right)$-linear in $X \in C^{\infty}\left(T_{\mathbb{C}} M_{\lambda, \Lambda}\right)$, the operator

$$
\nabla_{\Lambda W}^{\lambda}=\sum_{l=1}^{N} F_{l} \nabla_{\Lambda X_{l}}^{\lambda}
$$

is well defined. Let $W^{*} \in C^{\infty}$ (End $\left.\bar{\Lambda}\left(\mathscr{L}_{\Lambda}^{\lambda}\right) \otimes T_{\mathbb{C}}^{*} M_{\lambda, \Lambda}\right)$ be the dual of $W$ with respect to the metric $\hat{g}$; thus in local real coordinates $\mathbf{x}^{\mu}$ for $\mathbf{z}$

where

$$
W^{*}(\mathbf{z})=\sum_{l=1}^{N} \sum_{\mu} F_{l}(\mathbf{z}) \otimes X_{l}^{*}(\mathbf{z})_{\mu} d x^{\mu},
$$

$$
X^{*}(\mathbf{z})_{\mu}=\sum_{v} \hat{g}_{\mu v}(\mathbf{z}) X^{v}(\mathbf{z})
$$

Then for any $F_{0} \in C^{\infty}\left(\right.$ End $\left.\bar{\Lambda}\left(\mathscr{L}_{\Lambda}^{\lambda}\right)\right)$ we consider the SDE in the fibre of End $\bar{\Lambda}\left(\mathscr{L}_{\Lambda}^{\lambda}\right)$ over $\mathbf{z}=\mathbf{z}(0)$

$$
d U(t)=U(t)\left(\tilde{\tau}_{0}^{t} F_{0}(\mathbf{z}(t)) d t+W^{*}(\mathbf{z}(t)) \delta \mathbf{z}(t)\right)
$$

with initial condition $U(0)=$ id. Here again $\tilde{\tau}_{0}^{t}$ denotes the stochastic parallel transport in End $\bar{\Lambda}\left(\mathscr{L}_{\Lambda}^{\lambda}\right)$ along $\mathbf{z}(\cdot)$ of the fibre at $\mathbf{z}(t)$ onto the fibre at $\mathbf{z}$ such that a relation analogous to (5.18) holds. In (6.13) the multiplication of $W^{*}(\mathbf{z}(t))$ by the 
Itô differential $\delta \mathbf{z}(t)$ is given in local coordinates as

$$
\begin{aligned}
W^{*}(\mathbf{z}(t)) \delta \mathbf{z}(t)= & \sum_{l, \mu} \tilde{\tau}_{0}^{t} F_{l}(\mathbf{z}(t)) X_{l}^{*}(\mathbf{z}(t))_{\mu} \delta \mathbf{x}^{\mu}(t) \\
& -\frac{1}{2} \sum_{A, l, \mu, v} \tilde{\tau}_{0}^{t} F_{l}(\mathbf{z}(t)) L_{A}^{v}\left(\frac{\partial}{\partial \mathbf{x}^{\nu}} L_{A}^{\mu}\right)(\mathbf{z}(t)) X_{l}^{*}(\mathbf{z}(t))_{\mu} d t
\end{aligned}
$$

Note that

$$
\check{\Gamma}_{v \varrho}^{\mu}=-\sum_{A, \kappa} \hat{g}_{\varrho \kappa} L_{A}^{\kappa}\left(\frac{\partial}{\partial \mathbf{x}^{v}} L_{A}^{\mu}\right)
$$

are the Christoffel symbols of a connection $\check{V}$ (with nonvanishing torsion) on $T M_{\lambda, \Lambda}$ which is compatible with the metric $\hat{g}$. By construction the resulting Bochner Laplacian on $C^{\infty}\left(M_{\lambda, \Lambda}\right)$ equals $-\sum L_{A}^{2}$.

With the preceding constructions, there exists a unique solution of (6.13) satisfying the specified initial condition. In analogy to the discussion of Sect. 5, for $\varphi \in C^{\infty}\left(\bar{\Lambda}\left(\mathscr{L}_{\Lambda}^{\lambda}\right)\right)$ we obtain

$$
\left(\exp \left\{t\left(-\frac{1}{2} \hat{\Delta}+\nabla_{\Lambda W}^{\lambda}+F_{0}\right)\right\} \varphi\right)(\mathbf{z})=\mathbb{E}\left(U(t) \tau_{0}^{t} \varphi(\mathbf{z}(t))\right)
$$

In particular, if $W$ and $F_{0}$ are given by

and

$$
\begin{aligned}
W(\mathbf{z})= & i K_{h}(\mathbf{z})+J \sum_{\alpha, \beta} \sum_{\substack{k, k^{\prime} \\
k \neq k^{\prime}}}\left\{\left(Y_{\alpha}^{k}(\mathbf{z})+\mathbf{E}_{Y_{\alpha}^{k}}(\mathbf{z})\right) K_{Y_{\beta}^{k^{\prime}}}(\mathbf{z})\right. \\
& +\left(Y_{\beta}^{k^{\prime}}(\mathbf{z})+\mathbf{E}_{\left.Y_{\beta}^{k^{\prime}}(\mathbf{z})\right)} K_{Y_{\alpha}^{k}}(\mathbf{z})\right\} c^{\alpha \beta}\left(k, k^{\prime}\right)
\end{aligned}
$$

then

$$
\begin{aligned}
F_{0}(\mathbf{z})= & i \mathbf{h}(\mathbf{z})+i \mathbf{E}_{\mathbf{h}}(\mathbf{z})+\frac{1}{2} D \widetilde{\mathbf{K}}(\mathbf{z})-D \mathbf{S}(\mathbf{z})-\frac{1}{2} \operatorname{tr} \mathbf{S}_{\Lambda}(\mathbf{z}) \\
& +J \sum_{\alpha, \beta} \sum_{\substack{k, k^{\prime} \\
k \neq k^{\prime}}}\left(Y_{\alpha}^{k}(\mathbf{z})+\mathbf{E}_{Y_{\alpha}^{k}}(\mathbf{z})\right)\left(Y_{\beta}^{k^{\prime}}(\mathbf{z})-\mathbf{E}_{\left.Y_{\beta}^{k^{\prime}}(\mathbf{z})\right) c^{\alpha \beta}\left(k, k^{\prime}\right),}\right.
\end{aligned}
$$

$$
-\bar{\square}_{\Lambda}-H_{\Lambda}^{\lambda}=-\frac{1}{2} \hat{\Delta}+\nabla_{\Lambda W}^{\lambda}+F_{0} .
$$

Thus we have proved the following theorem.

Theorem 6.2. Let $U(t)$ be the solution of (6.13) for the choices (6.17) and (6.18). Then for all $\varphi \in C^{\infty}\left(\bar{\Lambda}\left(\mathscr{L}_{\Lambda}^{\lambda}\right)\right)$ and $t>0, \mathbf{z} \in M_{\lambda, \Lambda}$,

$$
\left(\exp \left\{-t\left(\bar{\square}_{\Lambda}+H_{\Lambda}^{\lambda}\right)\right\} \varphi\right)(\mathbf{z})=\mathbb{E}\left(U(t) \tau_{0}^{t} \varphi(\mathbf{z}(t))\right) .
$$

As in Sect. 5 we may now compare the resulting stochastic representation for the partition function with the representation in terms of the Euclidean Lagrange function of Sect. 4. Since this is straightforward, we refrain from giving the details.

\section{Classical Limits}

In this section we establish various classical limits in the purely bosonic sector employing methods developed in [Si, HPS, ST1].

We fix $\lambda$ and write $\bar{\square}=\bar{\square}^{\lambda}$ to exhibit the $\lambda$-dependence. The basic idea is to consider the irreducible representations $\pi_{n \lambda}$ as $n \rightarrow \infty, n=1,2,3, \ldots$ Recall that for $\lambda$, 
$\mu \in L \cap C$ the highest weight for the representation $\pi_{\lambda} \otimes \pi_{\mu}$ is given by $\lambda+\mu$ and the highest weight vector by $\psi_{\lambda} \otimes \psi_{\mu}$ (up to a scalar). This vector is hence contained in a copy of $\pi_{\lambda+\mu}$. Given $\lambda$, set

$$
\tilde{d}_{\lambda}=\prod_{\substack{\alpha \in \Delta^{+} \\\langle\lambda, \alpha\rangle \neq 0}} \frac{\langle\lambda, \alpha\rangle}{\langle\alpha, \varrho\rangle},
$$

where - as in Sect. $1-\varrho$ denotes half the sum of all the positive roots. Then with $d_{\lambda}=\operatorname{dim} \mathscr{H}_{\lambda}$, the dimension of the representation space $\mathscr{H}_{\lambda}$ of $\pi_{\lambda}$, and by virtue of Weyl's formula

$$
d_{n \lambda}=\widetilde{d}_{\lambda} n^{\operatorname{dim}_{\mathbb{C}^{M}}}(1+\mathcal{O}(1 / n)) .
$$

The first result of this section pertains to the one lattice point theory.

Proposition 7.1. The following classical limit relation holds for all $h \in \mathbf{g}$ :

$$
\begin{gathered}
\lim _{n \rightarrow \infty}\left(d_{n \lambda}\right)^{-2} \operatorname{Trace}_{L^{2}\left(\mathscr{L}^{n \lambda}\right)} \exp \left\{-t\left(\frac{1}{m} \bar{\square}^{n \lambda}-i d \pi_{0}^{n \lambda}\left(n^{-1} h\right)\right)\right\} \\
=\left(\tilde{d}_{\lambda}\right)^{-2} \int_{T^{*} M_{\lambda}} \exp \left\{-t H_{h, m}^{0, \mathrm{cl}}(p, z)\right\} d \mu_{T^{*} M_{\lambda}}(p, z) .
\end{gathered}
$$

Here $d \mu_{T^{*} M_{\lambda}}$ is the canonical volume form on the cotangent bundle $T^{*} M_{\lambda}$. We emphasize that $\mathscr{L}^{n \lambda}$ is regarded as a line bundle over the coadjoint orbit $M_{n \lambda}$. Also $M_{n \lambda}$ is equipped with the Riemannian metric induced by the Cartan-Killing form on $\mathbf{g}_{\mathbb{R}}^{*} \supset M_{n \lambda}$. In particular, the probability measure $d \mu_{M_{n \lambda}}(z)$ equals the canonical volume form on $M_{n \lambda}$ divided by the volume of $M_{n \lambda}$. The classical Hamiltonian $H_{h, m}^{0, \text { cl }}$ was defined by relation (4.7) in Sect. 4. Note that in the classical partition function the $p$-integration may be carried out such that (7.3) may be rewritten as

$$
\begin{aligned}
& \lim _{n \rightarrow \infty}\left(d_{n \lambda}\right)^{-2} \operatorname{Trace}_{L^{2}\left(\mathscr{L}^{n \lambda}\right)} \exp \left\{-t\left(\frac{1}{m} \bar{\square}^{n \lambda}-i d \pi_{0}^{n \lambda}\left(n^{-1} h\right)\right)\right\} \\
&=\left(\tilde{d}_{\lambda}\right)^{-2} \times \operatorname{Vol}\left(M_{\lambda}\right) \times\left(\frac{2 \pi m}{t}\right)^{\operatorname{dim}_{\mathbb{C}} M_{\lambda}} \\
& \quad \times \int_{M_{\lambda}} \exp \left\{t\left(i h(z)+\frac{m}{2}\left(K_{h}^{*}, K_{h}\right)(z)\right)\right\} d \mu_{M_{\lambda}}(z) .
\end{aligned}
$$

Moreover, in (7.3) and (7.4) the dependence on the $U(1)$-gauge potential $A$ has dropped out reflecting the absence of dia- and paramagnetism in the corresponding classical theory.

Proof of Proposition 7.1. We first observe that as subsets of $\mathbf{g}_{\mathbb{R}}^{*}$,

$$
M_{n \lambda}=n M_{\lambda} \text {. }
$$

Consider therefore the map

$$
n: M_{\lambda} \mapsto M_{n \lambda}, z \rightarrow n z .
$$

Under the pull-back by this diffeomorphism the line bundle $\mathscr{L}^{n \lambda}$ over $M_{n \lambda}$ goes into the line bundle $\left(\mathscr{L}^{\lambda}\right)^{\otimes n}$ over $M_{\lambda}$ such that the operators $Q_{n \lambda}$ turn into $\left(Q_{\lambda}\right)^{\otimes n}$ and $j_{n \lambda}$ into $\left(j_{\lambda}\right)^{\otimes n}$ (cf. Sect. 2). Also the probability measure $d \mu_{M_{n \lambda}}$ is mapped into $d \mu_{M_{\lambda}}$. 
Furthermore, by (2.42) the pull-back of the operator $\bar{\square}^{n \lambda}$ on $L^{2}\left(\mathscr{L}^{n \lambda}\right)$ is

$$
\frac{1}{2 n^{2}} \Delta^{\lambda, n}+\frac{1}{2 n^{2}} \operatorname{tr} S^{\lambda, n}
$$

on $L^{2}\left(\left(\mathscr{L}^{\lambda}\right)^{\otimes n}\right)$. Here $\Delta^{\lambda, n}$ is the Bochner Laplace operator on $\left(\mathscr{L}^{\lambda}\right)^{\otimes n}$ given by the connection $\nabla_{0}^{\lambda, n}=\left(Q_{\lambda}\right)^{\otimes n} d\left(j_{\lambda}\right)^{\otimes n}$, where we view $\left(\mathscr{L}^{\lambda}\right)^{\otimes n}$ as a subbundle of the trivial bundle $\mathscr{H}_{\lambda}^{\otimes n} \times M_{\lambda}$ over $M_{\lambda}$. Therefore, in a local gauge of $\mathscr{L}^{\lambda}$ (see Sect. 4) with real coordinates $x^{j}$ we have

$$
\Delta^{\lambda, n}=-\frac{1}{\sqrt{g}}\left(\partial_{j}+i n A_{j}\right) \sqrt{g} g^{j k}\left(\partial_{k}+i n A_{k}\right) .
$$

The curvature form $S^{\lambda, n}$ for $\nabla_{0}^{\lambda, n}$ satisfies $\operatorname{tr} S^{\lambda, n}=n \operatorname{tr} S^{\lambda}$. Finally, the pull-back of $-i d \pi_{0}^{n \lambda}(h / n)$ is given by

$$
-i h(z)+\frac{1}{i n} K_{h}(z)+\left(A, K_{h}\right)(z) .
$$

We are therefore in a situation covered by the discussion in [HPS, ST1] (see in particular Proposition 2.4 in [ST 1]) and the claim (7.3) follows. An analogous limit relation may be established on the space of $q$-forms using relation (2.91) in [ST1], but we refrain from providing details.

The discussion for the interacting case is analogous and we only give the results.

Proposition 7.2. For given $\lambda, \mathbf{c}$ and $\mathbf{h}$ let $m|J|$ be sufficiently small. Then

$$
\begin{aligned}
& \lim _{n \rightarrow \infty}\left(d_{n \lambda}\right)^{-2|\Lambda|} \operatorname{Trace}_{L^{2}\left(\mathscr{L}_{\Lambda}^{n \lambda}\right)} \exp \left\{-t\left(\frac{1}{m} \bar{\square}^{n \lambda}+H_{\Lambda}^{n \lambda}\left(n^{-2} J, \mathbf{c}, n^{-1} \mathbf{h}\right)\right)\right\} \\
& =\left(\tilde{d}_{\lambda}\right)^{-2|\Lambda|} \int_{T^{*} M_{\lambda, \Lambda}} \exp \left\{-t H_{J, \mathbf{c}, \mathbf{h}, m}^{0, \mathbf{c l}}(\mathbf{p}, \mathbf{z})\right\} d \mu_{T^{*} M_{\lambda, \Lambda}}(\mathbf{p}, \mathbf{z}),
\end{aligned}
$$

where $H_{A}^{n \lambda}$ was defined in Sect. 3 and $H_{A, J, \mathbf{c}, \mathbf{h}, m}^{0, \mathbf{c}}(\mathbf{p}, \mathbf{z})$ was defined by (4.20) in Sect. 4.

A similar relation can be derived if $H$ is replaced by $\tilde{H}$ [see (3.15)]. Note that the limit procedure $n \rightarrow \infty$ does not commute with the limit $m \rightarrow \infty$ in the purely bosonic sector as discussed in Sect. 2 and 3. In fact, with real $y^{\alpha}(k)=-i \mathbf{z}\left(Y_{\alpha}^{k}\right)=-i z(k)\left(Y_{\alpha}\right)$, our next proposition is a special case of Theorem 6.1 in [Si].

Proposition 7.3. The following classical limit relation holds:

$$
\begin{aligned}
& \lim _{n \rightarrow \infty}\left(d_{n \lambda}\right)^{-|\Lambda|} \operatorname{Trace}_{\mathscr{H}_{n \lambda}} \exp \left\{-t H_{\Lambda, n \lambda}\left(n^{-2} J, \mathbf{c}, n^{-1} \mathbf{h}\right)\right\}
\end{aligned}
$$

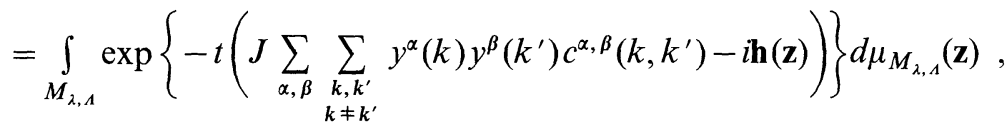

Moreover, an analogous relation holds if $H$ is replaced by $\tilde{H}$.

We conclude this section by specializing to the case $G=S U(2)$ and $\lambda$ defining the self-representation. Thus $\mathscr{H}_{\lambda}=\mathbb{C}^{2}$ and the highest weight vector $\psi_{\lambda}$ is $\left(\begin{array}{l}1 \\ 0\end{array}\right)$ if we 
choose as basis $Y_{\alpha}=i \sigma_{\alpha}$, where $\alpha=1,2,3$ and $i \sigma_{3}$ spans the Cartan subalgebra. Obviously $\lambda\left(Y_{\alpha}\right)=i \delta_{3 \alpha}$. Hence if again we let $y^{\alpha}=-i z\left(Y_{\alpha}\right)$ be the real coordinates of $z \in M_{\lambda}$ viewed as an element of $\mathbf{g}_{\mathbb{R}}^{*}$, we see that $M_{\lambda}$ is exactly the unit sphere $S^{2}$ in $\mathbf{g}_{\mathbb{R}}^{*} \cong \mathbb{R}^{3}$ with respect to the Euclidean norm $\|\cdot\|$.

Furthermore, the Killing vector fields $K_{Y_{\alpha}}$ on $M_{\lambda}$ are (up to a factor 2) the standard ones on $S^{2}$, i.e.

This gives

$$
K_{Y_{\alpha}}(z)=2 \sum_{\alpha, \beta=1}^{3} \varepsilon_{\alpha \beta \gamma} y^{\beta} \frac{\partial}{\partial y^{\nu}} .
$$

$$
\left(p, K_{Y_{\alpha}}(z)\right)=2(p \wedge y)^{\alpha},
$$

where $\wedge$ denotes the vector product and $T_{z}^{*} M_{\lambda}=T_{z}^{*} S^{2}$ is viewed as a subset of $\mathbb{R}^{3} \times \mathbb{R}^{3}$ such that $p \in T_{z}^{*} M_{\lambda}$ has Euclidean coordinates $p^{\alpha}$. Note that $p$ and $y$ are orthogonal. We recall the definition of $p^{2}$ as the length squared of $p$ with respect to the metric on $M_{\lambda}$ induced by the Cartan-Killing form $B$. The relation $b^{\alpha \beta}=-8 \delta^{\alpha \beta}$ for the inverse $b^{\alpha \beta}$ of $B\left(Y_{\alpha}, Y_{\beta}\right)$ follows from an easy calculation. Therefore $p^{2}=8\|p\|^{2}$.

Consequently, the classical Hamiltonian takes the form

$$
\begin{aligned}
& H_{A, J, \mathbf{c}, \mathbf{h}, m}^{0, \mathbf{c l}}(\mathbf{p}, \mathbf{z}) \\
& =\frac{8}{2 m}\|\mathbf{p}\|^{2}+J \sum_{\substack { \alpha, \beta \\
\begin{subarray}{c}{k, k^{\prime} \\
k \neq k^{\prime}{ \alpha , \beta \\
\begin{subarray} { c } { k , k ^ { \prime } \\
k \neq k ^ { \prime } } }\end{subarray}}^{3}(y(k)+2 p(k) \wedge y(k))^{\alpha}\left(y\left(k^{\prime}\right)+2 p\left(k^{\prime}\right) \wedge y\left(k^{\prime}\right)\right)^{\beta} c^{\alpha \beta}\left(k, k^{\prime}\right) \\
& \quad+\sum_{\alpha=1}^{3} \sum_{k \in \Lambda}(y(k)+2 p(k) \wedge y(k))^{\alpha} h^{\alpha}(k) .
\end{aligned}
$$

Here $\left\|\mathbf{p}^{2}\right\|=\sum_{k \in \Lambda}\|p(k)\|^{2}$, and we have written (in accordance with the notation of Sect. 1) $h(k) \in \mathbf{g}$ (the magnetic field $\mathbf{h}$ at $k \in \Lambda$ ) as $h(k)=\sum_{\alpha} h^{\alpha}(k) i \sigma_{\alpha}$ with $h^{\alpha}(k)$ real.

Finally we remark that for the particular choice

$$
c^{\alpha \beta}\left(k, k^{\prime}\right)=\left\{\begin{array}{ll}
-\delta^{\alpha \beta} & \text { if } k, k^{\prime} \text { N.N. } \\
0 & \text { otherwiese }
\end{array},\right.
$$

and using that $-i \mathbf{h}(\mathbf{z})=\sum_{\alpha} y^{\alpha}(k) h^{\alpha}(k)$, the right hand-side of (7.11) becomes the partition function of the classical isotropic Heisenberg (anti-)ferromagnet.

Acknowledgement. This work was supported in part by the Akademie der Wissenschaften zu Berlin.

\section{References}

[Bi1] Bismut, J.-M.: Mecanique aleatoire. Lecture Notes in Mathematics, Vol. 866. Berlin, Heidelberg, New York: Springer 1981

[Bi2] Bismut, J.-M.: The Atiyah -Singer theorems, a probabilistic approach. I. The index theorem. J. Funct. Anal. 57, 56-99 (1984) 
[Bi3] Bismut, J.-M.: Large deviations and the Malliavin calculus. Boston, Basel, Stuttgart: Birkhäuser 1984

[HMPS] Hogreve, H., Müller, W., Potthoff, J., Schrader, R.: A Feynman -Kac formula for the quantum Heisenberg ferromagnet. I. Commun. Math. Phys. 131, 465-494 (1990)

[HPS] Hogreve, H., Potthoff, J., Schrader, R.: Classical limits for quantum particles in external Yang -Mills potentials. Commun. Math. Phys. 91, 573-598 (1983)

[IW] Ikeda, N., Watanabe, S.: Stochastic differential equations and diffusion processes. Amsterdam, Oxford, New York: North-Holland 1981

[KN] Kobayashi, S., Nomizu, K.: Foundations of differential geometry, Vols. I and II. New York: Interscience 1963

[Ma] Malliavin, P.: Formule de la moyenne, calcul de perturbations et théorêmes d'annihilation pour les formes harmoniques. J. Funct. Anal. 17, 274-291 (1974)

[Me] Meyer, P.A.: Geometrie stochastique sans larmes. In: Séminaire de probabilitès. XV. Azèma, J., Yor, M. (eds.). Lecture Notes in Mathematics, Vol. 850, pp. 44-102. Berlin, Heidelberg, New York: Springer 1981

[Si] Simon, B.: The classical limit of quantum partition functions. Commun. Math. Phys. 71, 247-276 (1980)

[ST1] Schrader, R., Taylor, M.E.: Small $\hbar$ asymptotics for quantum partition functions associated to particles in external Yang-Mills potentials. Commun. Math. Phys. 92, 555-594 (1984)

Communicated by L. Alvarez-Gaumé

Received May 23, 1989 